\title{
Hope for pancreatic neuroendocrine tumors
}

\begin{abstract}
A
lthough pancreatic neuroendocrine tumors (NETs) are rare, their incidence and prevalence are increasing. In addition to this, most patients are diagnosed at a late stage of disease progression, with $65 \%$ of patients presenting with unresectable or metastatic disease. In patients with distant metastasis, median survival time is only 24 months. Unfortunately for these patients, at present there are only very limited treatment options and in the USA only streptozocin has been approved for the treatment of pancreatic NETs.

Two recent studies in this patient population seem to offer new therapy options for patients with this difficult-totreat disease. One study (the focus of this highlight) assessed the mTOR inhibitor everolimus, and the other study assessed the angiogenesis inhibitor sunitinib (to be discussed in an upcoming News \& Views).

James Yao, lead author of the everolimus study, described the background to the trial, "our initial reason for the
\end{abstract}

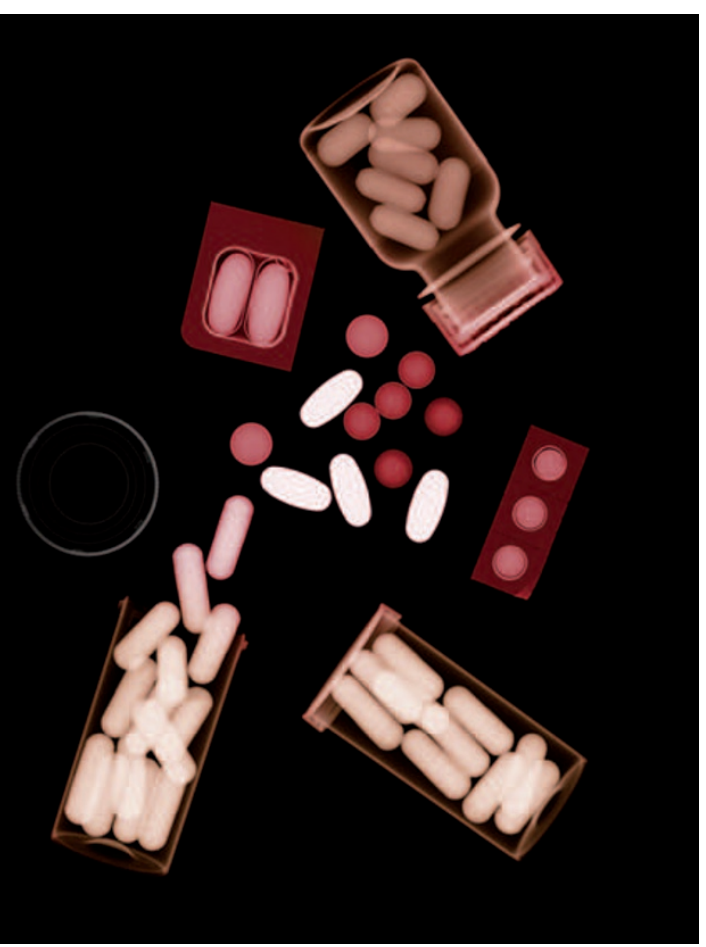

investigation of an mTOR inhibitor in NETs lies in the realization that genetic cancer syndromes in the mTOR pathway are associated with NETs." Furthermore, in two phase II studies in patients with pancreatic NETs, everolimus demonstrated promising antitumor activity. Yao continued, "more recently, somatic mutations in the mTOR pathway have also been identified."

The present study is an international, multicenter, double-blind, phase III trial that assessed best supportive care in addition to either everolimus (10 mg daily) or a matching placebo in 410 patients with pancreatic NETs and unresectable and/or metastatic disease. The primary end point of the study was progression-free survival. Yao explained the importance of the trial, "the study is a pivotal study designed to test the hypothesis that everolimus will delay tumor growth among patients with pancreatic NETs."

An important aspect of the study, which was also a limitation in the assessment of overall survival, is that owing to ethical considerations patients in the placebo arm whose disease progressed while on the trial were offered the chance to receive everolimus as an open-label treatment. This aspect of the protocol meant that there was no significant difference observed between the two arms in the secondary end point of overall survival. However, this end point will be reassessed once 250 deaths have occurred.

Despite this limitation, the primary end point of progression-free survival was significantly different between the two groups. Treatment with everolimus resulted in statistically significant and clinically relevant improvements in progression-free survival. The patients in the everolimus cohort had a median progression-free survival of 11.0 months and in those receiving placebo it was only 4.6 months. Yao went on to confirm that "the benefit was robust and seen across all prespecified subgroups," indicating that this is a treatment option for all patients with this disease, independent of age, previous treatment, race, geographic location, and tumor grade.

The difference in progression-free survival in the two groups seems to be caused by disease stabilization, rather than objective tumor responses. Confirmed tumor responses were observed in 10 patients receiving everolimus and in four patients receiving placebo.

In terms of safety, treatment with everolimus in this phase III study was consistent with the established profile observed from the phase II trials. Most of the adverse events were grade 1 or 2 ; however, one death in the everolimus group was related to the study drug.

The positive results from this study, and the concurrently published sunitinib trial, indicate that there might be several new options for patients with pancreatic NETs. The next steps for the researchers were summed up by Yao as "biomarkers and rational combinations." One such combination might be sunitinib and everolimus. In addition, Yao and colleagues are "currently conducting a phase I study of IMC A12, an insulin growth factor pathway inhibitor, in combination with everolimus.

In terms of biomarkers that might indicate efficacy of everolimus in patients with pancreatic NETs, somatic mutations were recently identified in the mTOR pathway among patients with pancreatic NETs. Yao and coworkers plan "to assess for mutations among patients treated with everolimus to identify predictive biomarkers." It is possible that these mutations could be used to identify responders to the therapy.

Rebecca Kirk 